\title{
99.9\% Spin-Flip Efficiency in the Presence of a Strong Siberian Snake ${ }^{1}$
}

HTML AESTRFICT + LINKS

V.S. Morozov" ${ }^{2 *}$ B.B. Blinov*, Z.B. Etienne ${ }^{3 *}$, A.D. Krisch*, M.A. Leonova*, A.M.T. Lin*, W. Lorenzon*, C.C. Peters*, D.W. Sivers", V.K. Wong* ${ }^{*}$, K. Yonehara*, V.A. Anferov ${ }^{\dagger}$, P. Schwandt ${ }^{\dagger}$, E.J. Stephenson ${ }^{\dagger}$, B. von Przewoski ${ }^{\dagger}$ and H. Sato**

${ }^{*}$ Spin Physics Center, University of Michigan, Ann Arbor, MI 48109-1120

${ }^{\dagger}$ Indiana University Cyclotron Facility, Bloomington, IN 47408-0768

${ }^{* *}$ KEK, High Energy Accelerator Research Organization, Tsukuba, Ibaraki 305-0801, Japan

\begin{abstract}
We recently studied the spin-flipping efficiency of an rf-dipole magnet using a 120-MeV horizontally polarized proton beam stored in the Indiana University Cyclotron Facility Cooler Ring, which contained a full Siberian snake. We flipped the spin by ramping the rf dipole's frequency through an rf-induced depolarizing resonance. By adiabatically turning on the rf dipole, we minimized the beam loss, while preserving almost all of the beam's polarization. After optimizing the frequency ramp parameters, we used up to 400 multiple spin flips to measure a spin-flip efficiency of $99.93 \pm 0.02 \%$. This result indicates that spin flipping should be possible in very-high-energy polarized storage rings, where Siberian snakes are certainly needed and only dipole rf-flipper magnets are practical.
\end{abstract}

\section{INTRODUCTION}

Polarized beam scattering experiments require frequent reversals in the beam polarization direction in order to reduce systematic errors in the measuredasymmetry. It is best to be able to spin-flip a stored polarized beam while it is circulating. Earlier we succesfully used an rf-solenoid to spin-flip a horizontally polarized proton beam stored in the Cooler Ring containing a Siberian snake with $97 \pm 1 \%$ spin-flip efficiency [1]. However, a solenoid's spin rotation decreases linearly with energy because of the Lorentz contraction of its $\int B d l$; thus, a solenoid is impractical for spin-flipping in high energy rings. Fortunately, a dipole's spin rotation is energy independent. Therefore, we recently studied an rf-dipole's ability to spin-flip a $120 \mathrm{MeV}$ horizontally polarized proton beam stored in the IUCF Cooler Ring operating with a full Siberian snake.

\footnotetext{
${ }^{1}$ Supported by research grants from the U.S. Department of Energy and the U.S. National Science Foundation

2 E-mail: morozov@umich.edu

3 Also at: Indiana University Cyclotron Facility, Bloomington, IN 47408-0768

${ }^{4}$ Also at: Portland Physics Institute, Portland, OR 97201, USA
} 


\section{SPIN MOTION AND SPIN FLIPPING}

In a storage ring, the beam's polarization vector precesses around the Stable Spin Direction with a frequency of

$$
f_{s}=f_{c} v_{s}
$$

where $f_{c}$ is the circulation frequency, and $v_{s}$ is the spin tune, which is the number of spin precessions during one turn around the ring. With a Siberian snake in the ring, the spin tune is given by

$$
v_{s}=\frac{1}{\pi} \cos ^{-1}[\cos (\pi G \gamma) \cos (\pi s / 2)]
$$

where $G=(g-2) / 2=1.792847$ is the proton's anomalous magnetic moment, $\gamma$ is its Lorentz energy factor, and $s$ is the snake strength. For a full (100\%) snake, $s=1$, and then $v_{s}=\frac{1}{2}$, independent of energy.

This spin motion can be perturbed by a horizontal rf magnetic field from either an rf-solenoid or an rf-dipole. Such perturbations can induce an rf depolarizing resonance, which can be used to flip the spin direction of the ring's stored polarized protons $[1,2]$. The resonant frequency $f_{r}$, at which an rf-induced depolarizing resonance occurs, is given by

$$
f_{r}=f_{c}\left(k \pm v_{s}\right),
$$

where $k$ is an integer. Sweeping the rf magnet's frequency through $f_{r}$ can flip the beam's spin direction. The Froissart-Stora equation [3] relates the beam's polarization after crossing the resonance $P_{f}$, to its initial polarization $P_{i}$,

$$
P_{f}=P_{i}\left\{2 \exp \left[\frac{-\left(\pi \varepsilon f_{c}\right)^{2}}{\Delta f / \Delta t}\right]-1\right\}
$$

where $\varepsilon$ is the resonance strength, and $\Delta f / \Delta t$ is the resonance crossing rate, while $\Delta f$ is the frequency ramp's range during the ramp time $\Delta t$. The resonance strength $\varepsilon$ is given by

$$
\varepsilon=\frac{\theta_{s}}{\pi}=\frac{G e \int B d l}{2 \pi m_{p} v},
$$

where $\theta_{s}$ is the rf-dipole's spin rotation angle, $m_{p}$ is the proton's mass and $v$ is its velocity. For a sufficiently strong resonance and low crossing rate, Eq. (4) indicates that $P_{f}$ is about equal to $-P_{i}$. The spin-flip efficiency $\eta$ is defined as

$$
\eta=\frac{-P_{f}}{P_{i}}
$$

\section{EXPERIMENTAL RESULTS}

The apparatus used for this experiment, including the rf-dipole, the IUCF Cooler Ring and the polarimeter were discussed earlier [1, 2]. The $120 \mathrm{MeV}$ horizontally polarized proton beam in the Cooler Ring was obtained using the new Cooler Injector Polarized 
IOn Source (CIPIOS) and the new Cooler Injection Synchrotron (CIS). The beam polarization was typically $77 \%$ after the $7 \mathrm{MeV}$ Linac and was practically the same at $120 \mathrm{MeV}$ injection into the Cooler Ring.

The circulation frequency in the Cooler Ring was $f_{c}=1.59784 \mathrm{MHz}$ at $120 \mathrm{MeV}$. With a nearly-full Siberian snake in the Ring, the spin tune $v_{s}$ is very near, but not exactly equal, to $\frac{1}{2}$. Thus, at $120 \mathrm{MeV}$,Eq. (3) implies that there should be two closely spaced $\mathrm{rf}$ spin resonances centered around $\frac{1}{2} f_{c}=0.79892 \mathrm{MHz}$, with their frequencies at $f_{r}^{-}=f_{c}\left(1-v_{s}\right)$ and $f_{r}^{+}=f_{c}\left(0+v_{s}\right)$. Since our snake strength was about 1.02 , the spin tune $v_{s}$ was about 0.510 ; thus, the $f_{r}^{-}$resonance should have a frequency slightly below $0.5 f_{c}$.

We first determined that the $f_{r}^{-}$resonance's frequency was near $0.777 \mathrm{MHz}$ by using our new resonance search technique:

- We first measured the beam polarization after sweeping the rf-dipole through some frequency range $\Delta f$, which might flip the spin.

- Then we cut the previous $\Delta f$ range into two equal halves and measured the polarization after sweeping the frequency through each half.

- Then we chose the $\Delta f$ range which caused spin-flip and repeated the process.

Fig. 1. shows the measured radial polarization plotted against each sweep's central frequency; the horizontal bars show the frequency range $\Delta f$ for each sweep. We then more precisely determined $f_{r}^{-}$and $f_{r}^{+}$by measuring the radial polarization at different fixed rf-dipole frequencies as shown in Fig. 2. Notice the very interesting behavior of the vertical polarization data in Fig. 2(b).

Next, we spin-flipped the beam by linearly ramping the rf-dipole's frequency from $f_{r}^{-}-5$ to $f_{r}^{-}+5 \mathrm{kHz}$, with various ramp times $\Delta t$, while measuring the beam polarization after each frequency ramp. The measured radial polarization is plotted against the ramp time in Fig. 3. This measured polarization is a good fit to the Froissart-Stora formula (Eq. (4)), which is shown by the curve.

To further study the spin-flip efficiency $\eta$, we next performed 10 spin-flips while varying the rf-dipole's frequency range $\Delta f$, its ramp time $\Delta t$ and its voltage $V$. The beam polarization after 10 spin-flips is plotted in Fig. 4 against the rf-dipole's voltage, with its $\Delta f$ and $\Delta t$ set to their optimum values. The dashed curve is a fit to the formula obtained by taking the $10^{\text {th }}$ power of $\eta \equiv-P_{f} / P_{i}$ in Eq. (4):

$$
P_{10}=P_{i} \cdot \eta^{10}=P_{i}\left\{2 \exp \left[\frac{-\left(\pi \varepsilon f_{c}\right)^{2}}{\Delta f / \Delta t}\right]-1\right\}^{10} .
$$

After maximizing the spin-flip efficiency by setting $\Delta t=27 \mathrm{~ms}, \Delta f= \pm 2 \mathrm{kHz}$ and $V=250 \mathrm{~V} \mathrm{rms}\left(\int B d l=0.43 \mathrm{~T} \cdot \mathrm{mm} \mathrm{rms}\right)$, we more precisely determined this efficiency $\eta$ by varying the number ofspin-flips; we measured the radial polarization after 0,100 , 200, 300 and 400 spin-flips while keeping $\Delta t, \Delta f$ and $V$ all fixed. This measured radial polarization is plotted against the number of spin-flips in Fig. $5^{5}$. We then fit these data using

$$
P_{n}=P_{i} \cdot \eta^{n}
$$

\footnotetext{
5 These data were obtained in a later run than the data in Figs. 1-4.
} 


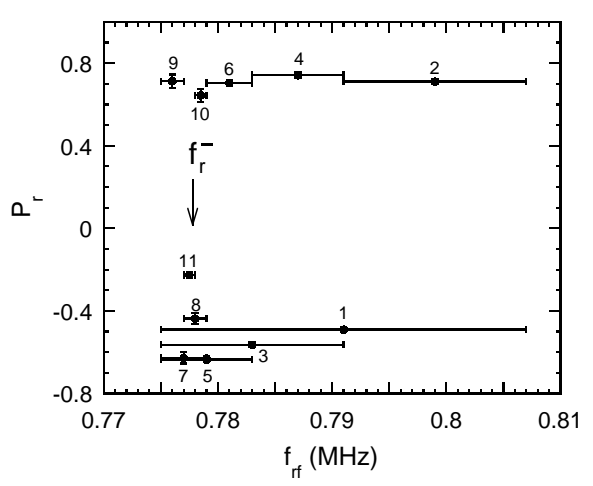

FIGURE 1. The measured radial proton polarization at $120 \mathrm{MeV}$ is plotted against the range of each frequency ramp; each frequency ramp's $\Delta f$ range is shown by a horizontal bar. The arrow shows $f_{r}^{-}$.

FIGURE 2. The measured radial (black) and vertical (white) proton polarizations at $120 \mathrm{MeV}$ are plotted against the rf-dipole's fixed frequency for the $f_{r}^{-}(a)$ and $f_{r}^{+}(b)$ resonances. The solid curves are fits using a secondorder Lorentzian. The arrows show $f_{r}^{-}$and $f_{r}^{+}$.
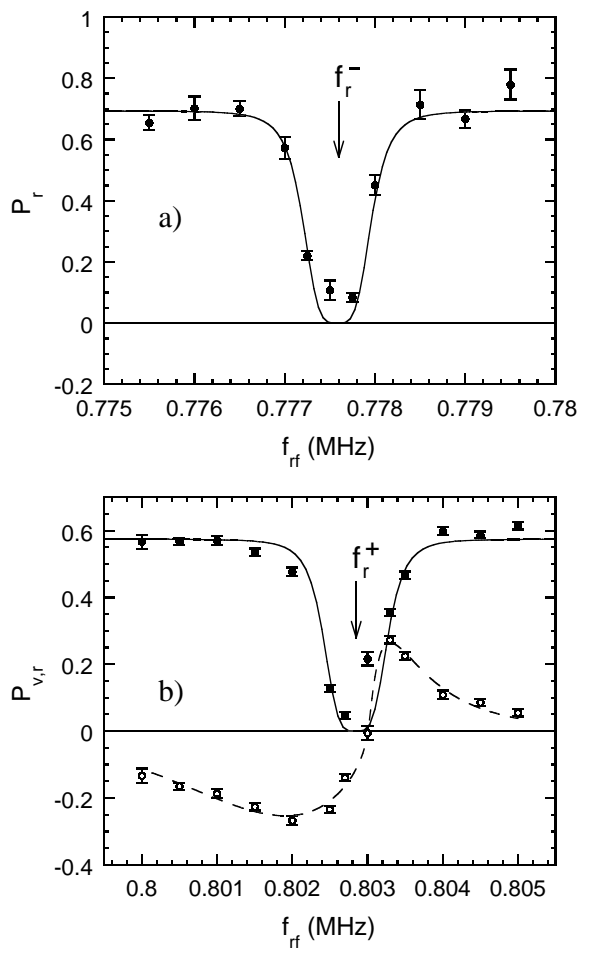

where $P_{n}$ is the measured radial beam polarization after $n$ spin-flips and $\eta$ is the spin-flip efficiency. The best fit gave a spin-flip efficiency of $99.93 \pm 0.02 \%$.

\section{ACKNOWLEDGMENTS}

We would like to thank the entire Indiana University Cyclotron Facility staff for the successful operation of the Cooler Ring.

\section{REFERENCES}

1. B.B. Blinov et al., Phys. Rev. Lett. 81, 2906 (1998);

V.A. Anferov et al., Proc. of 13th Intl. Symp. on High Energy Spin Physics, eds. N.E. Tyurin et al., World Scientific, 503 (1999).

2. B.B. Blinov et al., Phys. Rev. Lett. 88, 014801 (2002).

3. M. Froissart and R. Stora, Nucl. Instrum. and Methods 7, 297 (1960). 


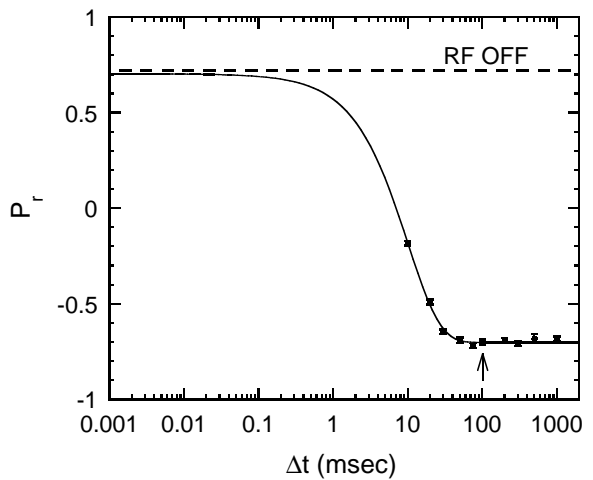

FIGURE 3. The measured radial proton polarization at $120 \mathrm{MeV}$ is plotted against the rf-dipole ramp time $\Delta t$. The curve is a fit using Eq. (4). The dashed line shows the polarization with the rfdipole off; the arrow shows the $100 \mathrm{~ms}$ setting used for later studies.

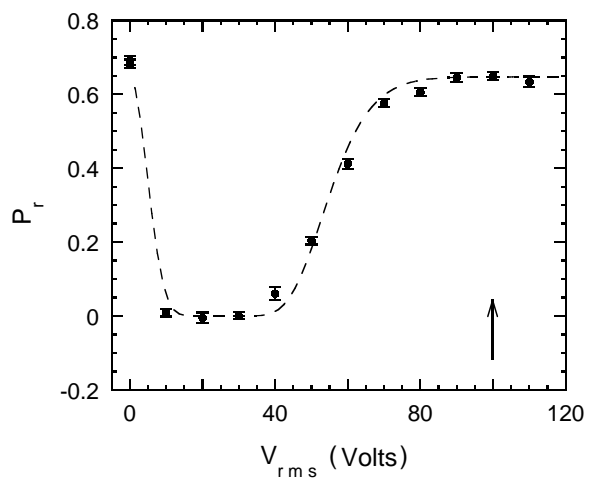

FIGURE 4. The measured radial proton polarization at $120 \mathrm{MeV}$ after 10 spin-flips is plotted against the rms rf-dipole voltage $V_{r m s}$. The dashed curve is a fit using Eq. (7); the arrow shows the $100 \mathrm{~V}$ setting used for later studies, which gives $\int B d l=0.17 \mathrm{~T} \cdot \mathrm{mm} \mathrm{rms}$.

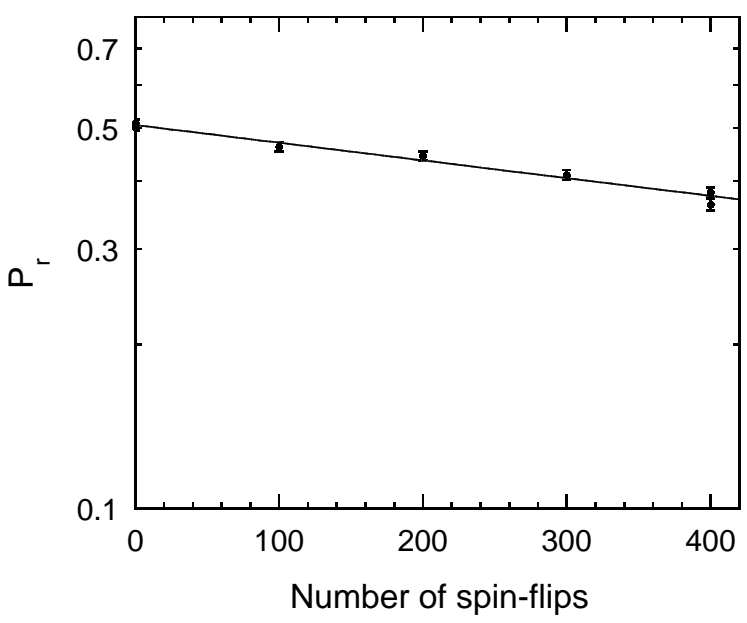

FIGURE 5. The measured radial proton polarization at $120 \mathrm{MeV}$ is plotted against the number of spin-flips. The curve is a fit to the data using Eq. (8). 